\title{
Central neurochemical ultradian variability in depression
}

\author{
Ronald M. Salomon ${ }^{\mathrm{a}, *}$, Benjamin W. Johnson ${ }^{\mathrm{b}}$ and Dennis E. Schmidt ${ }^{\mathrm{a}}$ \\ ${ }^{a}$ Department of Psychiatry, Vanderbilt University School of Medicine, Nashville, TN, USA \\ ${ }^{\mathrm{b}}$ Department of Anesthesiology, Vanderbilt University School of Medicine, Nashville, TN, USA
}

\begin{abstract}
Depression is characterized by blunted behavior and neuroendocrine function that generally improve with antidepressant treatment. This study examined intrinsic variability in brain neurotransmitter function, since it may be a source of blunted behavior and neuroendocrine function in depression and a marker for the illness, and has not previously been analyzed using wavelet decomposition. To measure variability in monoamine metabolites, lumbar cerebrospinal fluid (CSF) was collected in serial samples in depressed patients before and after treatment. We hypothesized that changes in variability would be observed after treatment. Mechanisms that control such variability may be critical to the pathophysiology of depression. Method: Time series data was obtained from serial ten-min sampling over a 24-hr period $(N=144)$ from thirteen depressed patients, with a repeat collection after 5 weeks of antidepressant (sertraline or bupropion) treatment. Concentrations of tryptophan (TRP), the monoamine metabolites 5-HIAA (metabolite of serotonin) and HVA (metabolite of dopamine), and the HVA:5HIAA ratio were transformed to examine power in slowly $(160 \mathrm{~min} / \mathrm{cycle})$ to rapidly $(20 \mathrm{~min} / \mathrm{cycle})$ occurring events. Power, the sum of the squares of the coefficients in each d (detail) wavelet, reflects variability within a limited frequency bandwidth for that wavelet. Pre-treatment to post-treatment comparisons were conducted with repeated measures ANOVA. Results: Antidepressant treatment was associated with increased power in the $\mathrm{d} 2$ wavelet from the HVA $(p=0.03)$ and the HVA:5-HIAA ratio $(p=0.03)$ series. The $\mathrm{d} 1$ and $\mathrm{d} 3$ wavelets showed increased power following antidepressant treatment for the ratio series $(\mathrm{d} 1, p=0.01 ; \mathrm{d} 3$, $p=0.05$ ). Significant changes in power were not observed for the 5-HIAA data series. Power differences among analytes suggest that the findings are specific to each system. Conclusion: The wavelet transform analysis shows changes in neurochemical signal variability following antidepressant treatment. Patterns or degrees of variability may be as important as, or possibly more important than, the mean levels of monoamine transmitters. Studies of variability observed in healthy individuals and a larger depressed sample will be needed to verify a relationship with mood and treatment response. Neurochemical measures of time-variability may be a pivotal marker in depression.
\end{abstract}

\section{Introduction}

Normal variations in mood over minutes to hours are an integral part of emotional stability. Brain neurochemical activity that underlies these variations must also vary within similar boundaries, but intrinsic variability in brain neurochemistry is rarely studied. We hypothesized that variability (power in wavelet transforms) would change with treatment. To test this, extended collections of cerebrospinal fluid (CSF) with

*Corresponding author: VAV Psychiatry-2200, 1500 21st Avenue S., Nashville, TN 37212, USA. Tel.: +1 615322 0387; Fax: +1 615 343 7868; E-mail: ron.salomon@ vanderbilt.edu. high frequency sampling of the serotonin precursor, tryptophan (TRP), and the serotonin and dopamine metabolites (5-HIAA and HVA, respectively) were explored for changes in their variability and interactions over time before and after treatment for depression.

Tryptophan (TRP) is an essential amino acid, derived solely from dietary protein, and passes the blood brain barrier via active transport throughout the neuraxis so that its levels in CSF will be influenced by dietary intake of TRP, hepatic homeostatic controls, and competition with other large neutral amino acids at the bloodbrain barrier. Serotonin is only one of many products of TRP metabolism, and is metabolized to 5-HIAA. Dopamine derives from tyrosine or phenylalanine, both of which are usually plentiful, and is metabolized to 
HVA. Metabolic turnover of the neurotransmitters is influenced by firing rates, synaptic release, reuptake activity, and exposure to catabolic enzymes. The ratio of HVA:5-HIAA, a previously published measure reflecting relative turnovers of the two neurotransmitters, was also determined $[9,15]$. Data suggest that the ratio may predict treatment outcome with greater fidelity than the estimates from either measure alone [15]. The present examination of the HVA:5-HIAA ratio in time series reflects, in a first approximation, the interactions and independence of serotonin and dopamine turnover. Flatter curves reflect greater correlation and dependence of these neurochemical systems, while greater variability reflects increasing independence of the two systems.

One way to determine whether variability in a neurochemical signal is a meaningful quantity is to monitor changes with treatment, to see if a consistent pattern is observed. Suitable data were available from an earlier study in which effects of TRP depletion were examined during the 25th through 48th hours of a CSF collection [18]. The first 24 hours of data had been collected while subjects maintained a balanced monoamine diet. A second, identical collection followed 5 weeks of antidepressant treatment. Changes in CSF HVA and 5HIAA levels, and the HVA:5-HIAA ratio during antidepressant treatment were examined within subjects in order to maximize the likelihood of detecting treatment effects on the variability of these measures.

The wavelet transform filters by frequency and location in the signal, giving a set of coefficients that quantify local variability power. Distinct features of wavelet transforms allow more sensitive detection of variability as irregular, not-quite-periodic events than can be obtained with other mathematical methods. Cosinor analysis estimates the degree of fit of a solitary waveform over the entire set (circadian, in most applications), and Fourier transforms detect a wide range of finely determined frequencies, but with poor temporal resolution and a strong vulnerability to phase shifts between events. Since ultradian variability in the brain is not necessarily locked into strict rhythms, phase shifts or complex cycling may mask quasi-periodic functions if analyses only use routine cosinor fits or Fourier spectral analyses. Wavelet transforms of serial neurochemical measures require a sampling frequency and duration relevant to neurobiological events in question, and highly reproducible neurochemical assays with limited, known noise characteristics. Similar wavelet transforms of neurophysiologic recordings following pharmacologic challenges have shown modulation of neural activity following ligand exposure in rodent cortex or globus pallidus [1,17].
Variability measures may lead to a better understanding of the pathophysiology of depression. Neurochemical variability characteristics in depression may be controlled, for example, by clock genes or by linkages between cell-cycle timing mechanisms and message transducers (e.g. groups of receptors). Altered cell timing mechanisms would be expected to simultaneously influence multiple neurochemical systems and their degree of interaction.

To summarize, levels of the monoamine metabolites HVA and 5-HIAA in serial samples of lumbar cerebrospinal fluid (CSF) taken every $10 \mathrm{~min}$ for $24 \mathrm{hrs}$ were analyzed with wavelet transforms to determine variability power or energy. Treatment-associated changes in these measures were assessed in a within-subjects design.

\section{Methods and materials}

Patients: CSF collection protocols were approved by the Vanderbilt Institutional Review Board. Each subject showed appropriate volition, motivation, and cognitive capacity, and discussed risks individually with the principal investigator at length before giving fully informed consent, signing the comprehensive consent document, and submitting to a thorough medical and psychiatric screening. Major depressive episodes were diagnosed using the Scheduled Clinical Interview for DSM-IV Axis I disorders (SCID-P) [10]. Severe personality disorders were excluded by SCID-II interview [11]. Diagnoses were confirmed in a lengthy clinical interview by a boarded psychiatrist (RMS). The Hamilton Rating Scale for Depression (HRSD) from the Yale Depression Inventory [14] was used to quantify depression severity. Patients were free of all psychotropic medications and substance abuse for two and six months respectively and were free of all other medications, including over-the-counter analgesics, for at least one month. Lifetime history of serious substance dependence was also cause for exclusion. Potentially suicidal patients were also excluded to avoid unethical delays in treatment, because of invasive study risks, and because of possibly inappropriate motivation for participation. Fifteen depressed patients (10 female, 5 male, ages 25 to 60 years, mean 37.7 years), met all above criteria and DSM-IV [3] criteria for Major Depressive Episode (Major Depressive Disorder or Bipolar Disorder, Depressed) and were admitted to the Vanderbilt General Clinical Research Center (GCRC). Data from 13 patients were analyzable. Data from one male was 
excluded because of a catheter occlusion. One female was excluded because she was maintained on lithium continuously and her data were extreme outliers, to be reported elsewhere.

Procedure: Details of the catheter placement and CSF collection procedure have been published elsewhere [18]. Briefly, a 72-hour pre-study caffeine-free and monoamine-balanced diet was continued during the collection period. During CSF collections, food was offered only at 6:30 a.m. and 9:30 a.m., with no snacks in between for a 21-hour fast. Hydration with intravenous 5\% dextrose was continuous. Patients remained supine overnight prior to and during CSF collections. Daytime napping was discouraged. Lights were out from 10:30 p.m. to 6:00 a.m. Tympanic temperature was determined every $2 \mathrm{hrs}$.

Catheter placement began at 7:00 a.m. A 20g epidural catheter at L4-5 connected to a peristaltic pump collected $1 \mathrm{ml}$ samples every $10 \mathrm{~min}(0.1 \mathrm{ml} / \mathrm{min})$ for $24 \mathrm{hrs}$ beginning at 8:00 a.m. Samples were chilled to $5^{\circ} \mathrm{C}$. during collection, moved to dry ice storage within $30 \mathrm{~min}$, and stored at $-70^{\circ} \mathrm{C}$. HPLC analysis with electrochemical detection [20] determined concentrations of the amino acid precursor of serotonin (tryptophan, TRP), the serotonin metabolite (5hydroxyindoleacetic acid, 5-HIAA), and the dopamine metabolite (homovanillic acid, HVA).

The procedure was repeated after five weeks of treatment. Moderate to severe headaches occurred after half of the procedures, which were otherwise well tolerated. All headaches resolved, with blood patches performed for nine of the 30 studies. No serious adverse events or long-term sequellae resulted from this study.

Antidepressant treatment: Eight weeks of sertraline treatment (100 mg daily at 10:00 a.m.) or bupropion treatment (300 mg daily at 10:00 a.m.) began with halfdoses during week one. Five patients were studied under a sertraline-only protocol, the following ten patients were assigned to drug in random fashion. Data was collected in conjunction with another study for which the random assignment to treatment was a critical feature and fortuitously provided evidence of changes in variability power irrespective of antidepressant mechanism of action, but limited the sample size for each treatment.

Analysis: Time series data were examined for treatment-associated changes in means, variance, and wavelet coefficient power. Results were compared to Fourier-derived spectral analysis findings recently published elsewhere [19]. The present analysis using wavelet properties was conceived and executed after study completion and analysis with Fourier methods.
Wavelet transforms (Fig. 1) characterize abrupt changes in signals, localizing power in bandwidths of event proximities, conserving orthogonal and power properties relevant to statistical analyses, and backtransforming faithfully. Transform parts, called "a", and "d", are calculated in an iterative process. Each iteration, $m$, smoothes the signal with a local wavelet convolution (similar to a low-pass filter) and halves the signal length. This new "a" becomes shorter with each level of the transform. At the same time, a " $\mathrm{d}_{\mathrm{m}}$ " wavelet is appended, maintaining the original signal length, and representing local abrupt discontinuities (changes) in the signal (level one) or previous " $a$ ". The local convolution of 4 sequential points with the wavelets to form " $\mathrm{d}_{\mathrm{m}}$ " demonstrates progressively coarser variability in the original signal. Energy (sum of squares) in $d_{1}$ is mostly from fine variability (events every 20 to $40 \mathrm{~min}$ ), $\mathrm{d}_{2}$ reflects mid-frequency variability (40 to $80 \mathrm{~min}$ between events), and $\mathrm{d}_{3}$ reflects slower variability (about 80 to 160 min event intervals).

Raw signals (13 paired collections of $N=144$ points each, all beginning at 8 am and spanning 24 hours) were transformed to level 3 using the Daubechies 2 wavelet transform [8] using a custom-written program for MATLAB (The Mathworks, Inc., Natick, MA). Resulting $\mathrm{d}_{1}, \mathrm{~d}_{2}$, and $\mathrm{d}_{3}$ wavelets were segmented to represent daytime (s1, 8 a.m. to 4 p.m.), evening (s2, 4 p.m. to 12 midnight), and nighttime (s3: 12 midnight to 8 a.m.) time periods. The power (sum of squares of the $d$ coefficients in the time segment) was calculated for each of these nine time segments (wavelet $\times$ 'time of day').

Main effects and interactions of time segment and treatment condition were analyzed using repeated measures analysis of variance (ANOVA) tests. Time segment (morning/afternoon/evening) and treatment condition (pre-treatment/post-treatment) were both withinsubjects factors in this doubly repeated model. All statistics are two-tailed with $\alpha=0.05$, and means are reported as mean \pm standard deviation. Significant findings were followed by post-hoc analyses, for which alpha was adjusted for multiple comparisons (e.g. 0.025 for main effect of segment). Analyses were performed using the SAS statistical package (version 8.1 SAS Institute Inc., Cary, NC).

Quality assurance procedure: A comparison of results among the four (5-HIAA, TRP, HVA, and ratio) time series was performed to exclude artifacts due to shared signal properties. Wavelet profiles (Fig. 1) and the power values from them differed among the four chemical time-series traces studied by HPLC and also 

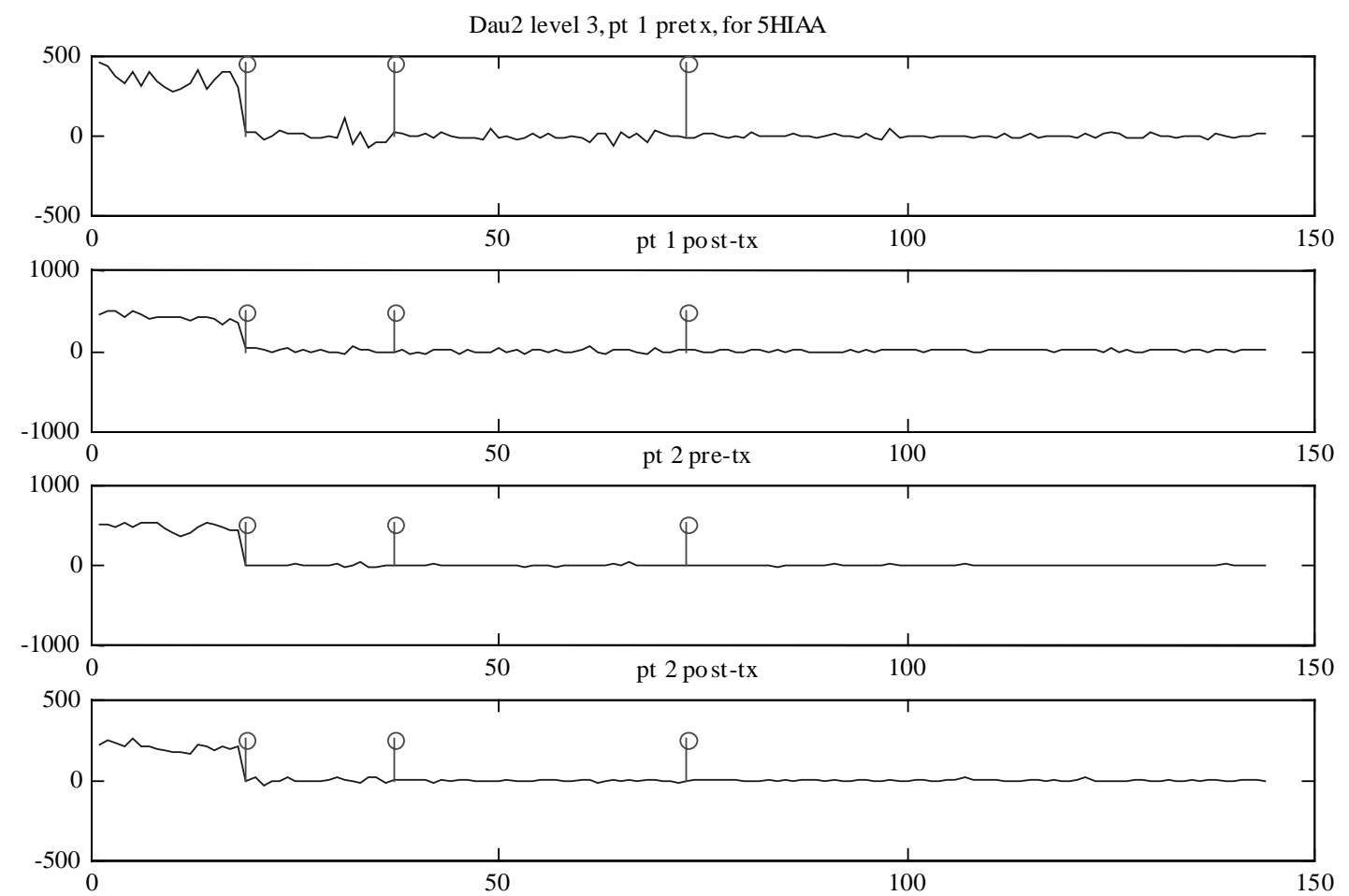

Fig. 1. Examples of wavelet transforms from 5-HIAA concentrations serially collected before and after treatment from two patients with depression. Stem markers delineate the "a" signal transform, followed by the "d3", "d2", and "d1" wavelets respectively. To a first approximation, power in the $\mathrm{d} 1$ wavelet represents power from finer variations at frequencies of one cycle per 20 to $40 \mathrm{~min}$. The $\mathrm{d} 2$ and $\mathrm{d} 3 \mathrm{wavelets}$ reflect power from variance with 40 to $80 \mathrm{~min}$ and 80 to $160 \mathrm{~min}$ periods respectively. Each d wavelet was subdivided (not shown) into three time segments corresponding to day ( 8 a.m. to 4 p.m.), evening ( 4 p.m. to $12 \mathrm{MN}$ ), and night (12 MN to 8 a.m.) for analyses described in the text.

for other neuropeptide measures from the same samples (data not shown), strongly supporting the contention that the observed variability is not related to a common artifact in sample collection or biochemical analysis methods.

\section{Results}

5-HIAA. One interaction term between treatment condition and time segment in the $\mathrm{d} 3$ wavelet nearly reached significance $(F(2,11)=3.64, p=0.06$, Fig. 2a). None of the post-hoc comparisons reached significance and there were no main effects.

Tryptophan. Peak variability was anticipated following mealtimes, but was observed instead during fasting periods. Time of day affected TRP d3 power $(F(2,11)=7.74, p=0.01$, Fig. $2 b)$, but none of the time segment pairs differed significantly. The difference appeared to be primarily in a lower daytime power compared to evening and nighttime (daytime $2.3 \mathrm{e} 5 \pm$ $1.5 \mathrm{e} 5$, evening $3.9 \mathrm{e} 5 \pm 4.0 \mathrm{e} 5$, night $3.7 \mathrm{e} 5 \pm 2.0 \mathrm{e} 5)$.
Meals were given at 6:30 a.m. and 9:30 a.m., possibly suggesting that variability may be enhanced while fasting.

$H V A$. Antidepressant treatment was associated with increased power in the $\mathrm{d} 2$ wavelet from the HVA series $(F(1,12)=6.11, p=0.03$, Fig. 2c). The HVA series showed an effect of the time period (day, evening, or night, irrespective of treatment status) on variability power $(\mathrm{d} 1: F(2,11)=4.62, p=0.04$, $\mathrm{d} 2: F(2,11)=7.03, p=0.01$, and $\mathrm{d} 3: F(2,11)=$ $6.90, p=0.01)$.

We performed pairwise posthoc comparisons of the time segment averages (across treatment conditions) for each of the three wavelets to explore the main effects of time segment in HVA. Power in the evening (s2) was greater than daytime (s1) for the d1 wavelet of the HVA signal $(F(1,12)=6.94, p=0.02)$. The nighttime (s3) power was greater than the evening (s2) for two wavelets: $\mathrm{d} 2(F(1,12)=6.62, p=0.02)$ and $\mathrm{d} 3$ $(F(1,12)=8.91, p=0.02)$.

HVA:5-HIAA ratio. Antidepressant treatment was associated with increased power in the ratio $\mathrm{d} 1$ 

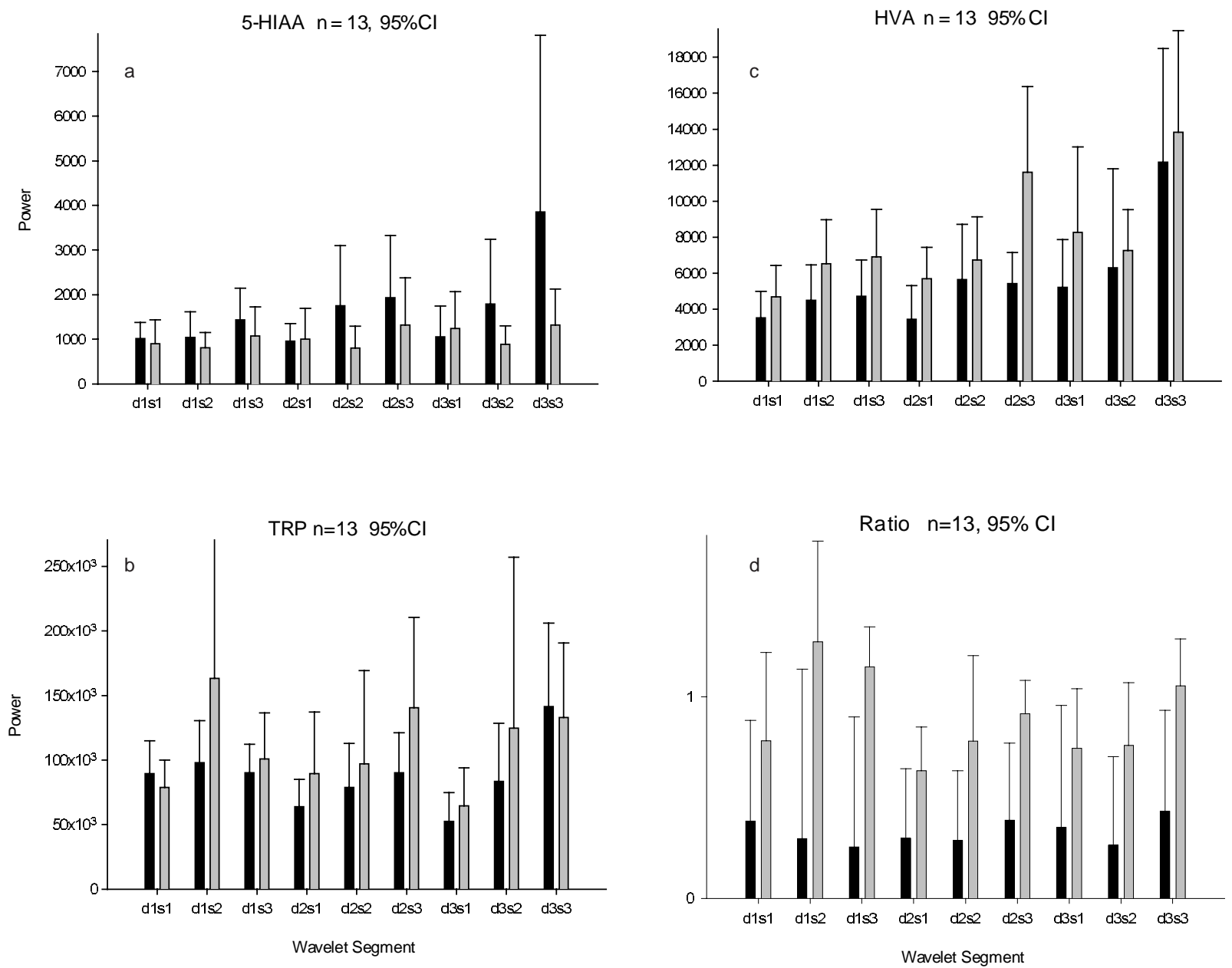

Fig. 2. Mean time segment power from Daubechies 2 wavelet transforms of (a) 5-HIAA series, (b) TRP series, (c) HVA series, and (d) 5-HIAA:HVA ratio series from depressed patients before and after antidepressant treatment. Significant differences discussed in text. $(n=13$, mean $95 \%$ confidence interval).

$(F(1,12)=9.12, p=0.01$, Fig. $2 \mathrm{~d}), \mathrm{d} 2(F(1,12)=$ $5.70, p=0.03)$, and $\mathrm{d} 3(F(1,12)=4.72, p=0.05)$ wavelet series. These effects were observed evenly across most subjects (Fig. 3). This finding strongly supports the hypothesis that treatment would be associated with changes in variability in monoamine system interactions.

\section{Discussion}

Mechanisms that regulate CNS neurochemical variability may be involved in the pathophysiology of depression. We report here that variability in neurochemical signals changed significantly after treatment with antidepressant medications for five weeks, with most remarkable increases in the variability of the ratio of serotonin to dopamine metabolites. This may imply that healthy moods are associated with a degree of independence and autonomy of monoaminergic systems that is diminished in depression. Variability was determined by as power in the wavelet transforms of serotonin and dopamine metabolite levels in q10 min, serial CSF samples collected for 24 hours. The finding suggests that variability carries information that is not included in measures of mean activity or levels, and that may be relevant to depression pathophysiology and treatment. The 'stuckness' of neurochemical activity may be a predictor of the 'stuckness' of moods.

Greatest treatment-associated change in variability was observed in the signal generated from the ratio of HVA:5-HIAA, where a consistent and sizeable increase in power was observed across wavelets and through 
Ratio d3s3 Power by Patient

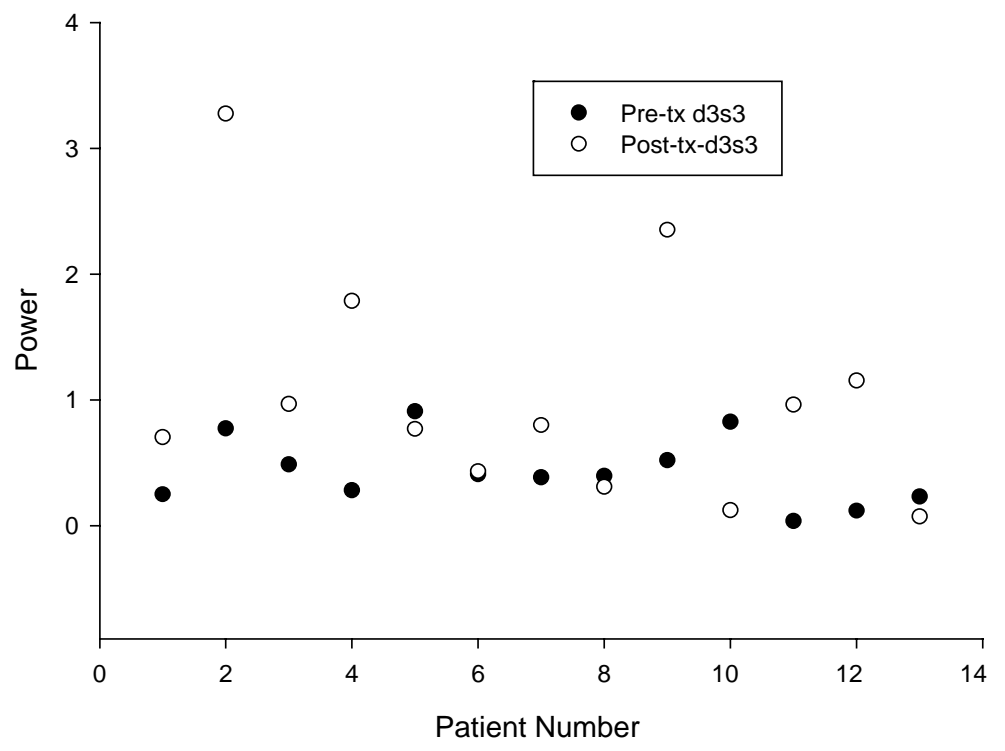

Fig. 3. Power in $\mathrm{d} 3$ nighttime (s3) segments by patient before and after treatment. (paired $t(12)=-2.463, p=0.03$ ).

each of the daytime segments. This appears to indicate increased independence of these monoaminergic systems, and was strongest for high frequency events (20-40 min intervals) in the evening (4 p.m. to midnight) and nighttime (midnight to 8 a.m.) segments. The synchrony of these systems prior to treatment may represent a baseline deficit in independence, or it may be that the increased independence after treatment represents a treatment effect unrelated to antidepressant mechanisms of action. Healthy control data is being collected in the same manner in order to begin to address this question. However, it is notable that both antidepressants, one with serotonergic and the other with catecholaminergic activity, were associated with similar enhancements of independence of the transmitter turnover signals.

Individually, variability power in the signal from the serotonin metabolite, 5-HIAA, trended towards a decline after treatment. A larger sample size might reveal that treatment suppresses variability in the nighttime turnover of central serotonin, or alternatively, that serotonin turnover variability is enhanced in depression. This is consistent with observations of REM suppression during antidepressant treatment, putatively related to decreased variability at night. Direct correlations with REM suppression are needed to validate this supposition.

At the same time, variability of TRP (the serotonin precursor) levels in CSF was weakest in the daytime (compared to evening and nighttime), with no significant change related to treatment. This may suggest that the TRP supply from the bloodstream is more stable around mealtimes, and also indicates that variability in TRP, the serotonin precursor, was not directly related to variability in the metabolite of serotonin.

The variability of the dopamine metabolite, HVA, increased significantly in power in the mid-frequency (40-80 min/cycle) after treatment. Most of this treatment-associated increase was observed at night in this mid-frequency bandwidth, contrasting with the decline in 5-HIAA at slower frequencies that also appeared at night.

These wavelet transform analyses support the hypothesis that variability in neurochemical activity changes with treatment, as was previously suggested using Fourier transforms ([19], under review). Differences in the findings are of interest. Fourier analyses detect only strictly rhythmic patterns that must be locked in phase over the analyzed time segment, while the wavelet transform is more sensitive to local singular, closely linked, or irregularly occurring events. Both analyses suggest that post-treatment enhancement in variability in the HVA:5-HIAA ratio may be a response marker.

These studies remain preliminary and will require replication. Between-subjects variables (age, gender, diagnosis and subtype, baseline mood severity, treat- 
ment used, treatment response) were not included in this preliminary analysis due to the small number of subjects $(n=13)$. In part, the use of two treatments and the diversity of diagnostic subtypes deliberately allowed detection of a putatively universal feature of depression, diminished variability in neurochemical systems. Also, a study of prolonged CSF collection with absolutely strict diagnostic inclusion criterion would be impossible to complete because of recruitment challenges. Replication and extension studies will need to show subgroup differences and shared features, and identify features that predict treatment preferences, treatment resistance, and high mortality.

A diminished neurochemical variability model reported here fits existing data on the neurobiology of depression, and may explain the pathophysiology and psychopathology of depression subtypes. Ultradian components of variability are important since, in healthy individuals, subtle but appreciable mood changes frequently occur over the course of minutes to hours. For example, consider the range of moods that most people experience while watching sports events or theater presentations. Contrasts between depression and health may lie in the diminished recruitment and utilization of healthy fluctuations in mental processing.

\section{Brief comment on dynamic neurochemical models of depression}

The present dynamic neurochemical analyses contrast with metrics that treat behavior, brain physiology, and transmitter neurochemistry as static conditions, observed over time but expressed as a mean with a population variance. Dynamic measures of motor [5] and social activity [21], and also of plasma tryptophan levels [7] have shown diminished variability in depression. Rhythms in actigraphy are also blunted in childhood and adolescent depression [4]. Activity phases relative to time of day and duration of wakefulness are independent, but non-additive factors controlling subjective reports of mood in healthy subjects [6]. Neuroendocrine responses also show ultradian variability, but altered central monoamine variability has not been shown previously.

In melancholia, more frequent and more lethal suicide attempts [13], represent diminished variability in thought processes and central neurochemistry. Melancholia is more consistently associated with disrupted anterior cingulate cortical function than other depression subtypes [16] and with a defective intracellular linkage through second messenger systems coupled to the serotonin-2a receptor [2] that would be expected to functionally disrupt cellular variability.

\section{Conclusions}

Neurochemical variability changes during antidepressant treatment, possibly reflecting a mood-determining process. Alternatively, this finding may be an artifact of treatment, not associated with mood pathophysiology, but other findings in behavioral or peripheral monoaminergic markers are consistent with an interpretation that the present findings represent truly altered dynamics of central monoaminergic function in depression. Most notably, the HVA:5HIAA ratio variability increased significantly, suggesting an increase in independence between the two monoamine systems with treatment.

Variability was determined over time intervals relevant to mood behaviors. At a minimum, these analyses suggest that further study is warranted to ascertain whether variability in neurobiological activity in depression can be used as a general marker in depression. The main limitations in this study were the small number of subjects and the absence of healthy control comparison data, which are in turn related to the invasiveness of the procedure. Although invasive methods may be justified by the severe morbidity and mortality caused by depression, general application of the lessons from these studies will require the development of less invasive methods of detection of central neurochemical rhythms.

In the interim, further clarification of variability characteristics in neurochemical measures from CSF will be needed to design imaging or other methods to detect neurochemical variability using less invasive measurement methods. Monoaminergic systems display ultradian variability (and other systems are likely to, as well), but most of the currently available brain imaging methods measure regionally global activity that is not specific to single neurochemical systems. Detection and descriptions of spontaneous variability characteristics in brain functions may allow an identification of homogeneous populations with similar disturbances of neurochemical variability, and then permit investigations into genetic regulators of spontaneous variability.

\section{Acknowledgements}

The authors are grateful to Douglas Hardin, Ph.D., Associate Professor of Mathematics, Vanderbilt University, and to Elizabeth Stringer and Reuben Fan for guidance and assistance with mathematical analyses. Statistical assistance from Jennifer Urbano Blackford, 
Ph.D., Vanderbilt Kennedy Center for Research on Human Development (NICHD Grant P30 HD15052) is gratefully acknowledged. This work was funded by grants to RMS (K23 MH01828, the Stanley Foundation, NARSAD, and an investigator-initiated grant from Pfizer, Inc.) and to the Vanderbilt General Clinical Research Center (M01 RR00095).

\section{References}

[1] M. Akay, Y.M. Akay, P. Cheng and H.H. Szeto, Investigating the effects of opioid drugs on electrocortical activity using wavelet transform, Biol Cybern 72 (1995), 431-437.

[2] D. Akin, D.H. Manier, E. Sanders-Bush and R.C. Shelton, Decreased Serotonin 5-HT(2A) Receptor-Stimulated Phosphoinositide Signaling in Fibroblasts from Melancholic Depressed Patients, Neuropsychopharmacology 29 (2004), 2081-2087.

[3] American Psychiatric Association, Diagnostic and Statistical Manual of Mental Disorders, (Fourth ed.), Washington, DC, APA, 1994.

[4] R. Armitage, R. Hoffmann, G. Emslie, J. Rintelman, J. Moore and K. Lewis, Rest-activity cycles in childhood and adolescent depression, J Am Acad Child Adolesc Psychiatry 43 (2004), 761-769.

[5] O. Benoit, S. Royant-Parola, A.A. Borbely, I. Tobler and D. Widlocher, Circadian aspects of motor activity in depressed patients, Acta Psychiatr Belg 85 (1985), 582-592.

[6] D.B. Boivin, C.A. Czeisler, D.J. Dijk, J.F. Duffy, S. Folkard, D.S. Minors, P. Totterdell and J.M. Waterhouse, Complex interaction of the sleep-wake cycle and circadian phase modulates mood in healthy subjects, Arch Gen Psychiatry 54 (1997), 145-152.

[7] M. Candito, D. Pringuey, A. Iordache, E. Souetre, P. Chambon and G. Darcourt, Circadian variation in total plasma tryptophan. Antidepressant treatment: drugs and phase advance, Life Sci 50 (1992), PL71-74.

[8] I. Daubechies, Ten Lectures on Wavelets, SIAM, Philadelphia, PA, 1990.

[9] G. Engstrom, C. Alling, K. Blennow, G. Regnell and L. Traskman-Bendz, Reduced cerebrospinal HVA concentrations and HVA:5-HIAA ratios in suicide attempters. Monoamine metabolites in 120 suicide attempters and 47 controls, Eur Neuropsychopharmacol 9 (1999), 399-405.

[10] M.B. First, R.L. Spitzer, M. Gibbon and J.B.W. Williams, Structured Clinical Interview for DSM-IV Axis I Disorders (SCID-I/P), (Version 2.0), Biometrics Research Department,
New York State Psychiatric Institute, 722 West 168th Street, NY 10032, 1996.

[11] M.B. First, R.L. Spitzer, M. Gibbon, J.B.W. Williams and L. Benjamin, Structured Clinical Interview for DSM-IV Axis II Personality Disorders (SCID-II), (Version 2.0), Biometrics Research Department, New York State Psychiatric Institute, 722 West 168th Street, NY 10032, 1996.

[12] K.N. Fountoulakis, A. Iacovides, P. Grammaticos, G. St. Kaprinis and P. Bech, Thyroid function in clinical subtypes of major depression: an exploratory study, BMC Psychiatry 4 (2004), 6.

[13] M.F. Grunebaum, H.C. Galfalvy, M.A. Oquendo, A.K. Burke and J.J. Mann, Melancholia and the probability and lethality of suicide attempts, Br J Psychiatry 184 (2004), 534-535.

[14] C. Mazure, J.C. Nelson and L.H. Price, Reliability and Validity of the Symptoms of Major Depressive Illness, Arch Gen Psychiatry 43 (1986), 451-456.

[15] E.D. Risby, J.K. Hsiao, T. Sunderland, H. Agren, M.V. Rudorfer and W.Z. Potter, The effects of antidepressants on the cerebrospinal fluid homovanillic acid:5-hydroxyindoleacetic acid ratio, Clin Pharmacol Ther 42 (1987), 547-554.

[16] M.A. Rogers, M.A. Bellgrove, E. Chiu, C. Mileshkin and J.L. Bradshaw, Response selection deficits in melancholic but not nonmelancholic unipolar major depression, J Clin Exp Neuropsychol 26 (2004), 169-179.

[17] D.N. Ruskin, D.A. Bergstrom, D. Baek, L.E. Freeman and J.R. Walters, Cocaine or selective block of dopamine transporters influences multisecond oscillations in firing rate in the globus pallidus, Neuropsychopharmacology 25 (2001), 28-40.

[18] R.M. Salomon, J.S. Kennedy, B.W. Johnson Jr., D.E. Schmidt, J. Kwentus, H.E. Gwirtsman and M.H. Ebert, Association of a critical CSF tryptophan threshold level with depressive relapse, Neuropsychopharmacology 28 (2003), 956-960. Epub 2003 Mar 19.

[19] R.M. Salomon, J.S. Kennedy, B.W. Johnson Jr., J.U. Blackford, D.E. Schmidt, J. Kwentus, H.E. Gwirtsman, J.F. Gouda and R.G. Shiavi, Treatment enhances ultradian rhythms of CSF monoamine metabolites in patients with major depressive episodes, Neuropsychopharmacology, Epub 2005, ahead of print April 27.

[20] D.E. Schmidt, M.L. Roznoski and M.H. Ebert, Qualitative and quantitative HPLC analysis of monoamine neurotransmitters and metabolites in CSF and brain tissue using reductive electrochemical detection, Biomedical Chromotography 4 (1990), 215.

[21] M.P. Szuba, A. Yager, B.H. Guze, E.M. Allen and L.R. Baxter Jr., Disruption of social circadian rhythms in major depression: a preliminary report, Psychiatry Res 42 (1992), 221-230. 


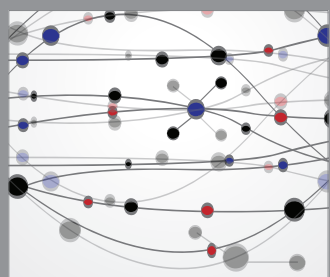

The Scientific World Journal
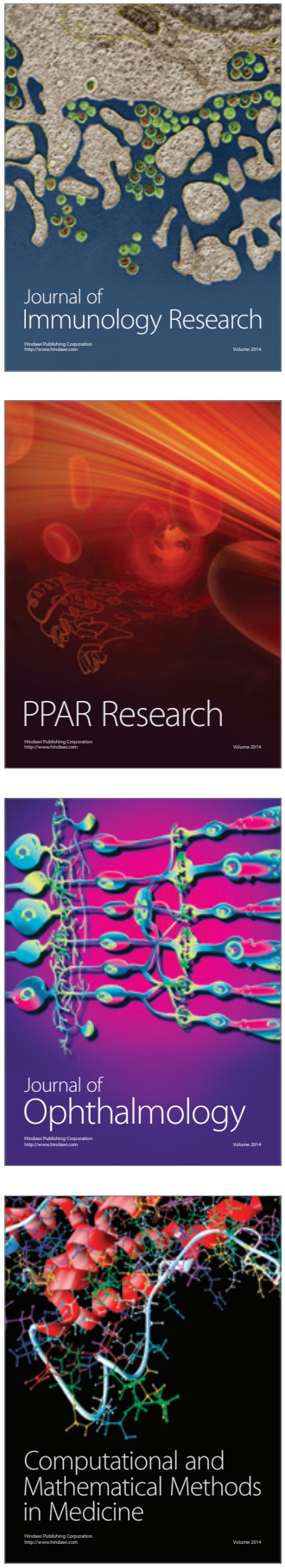

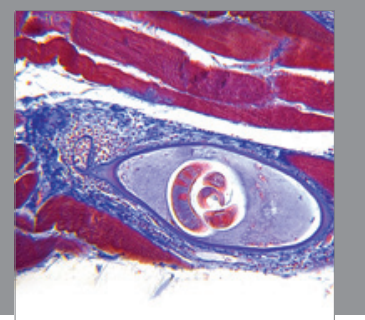

Gastroenterology

Research and Practice
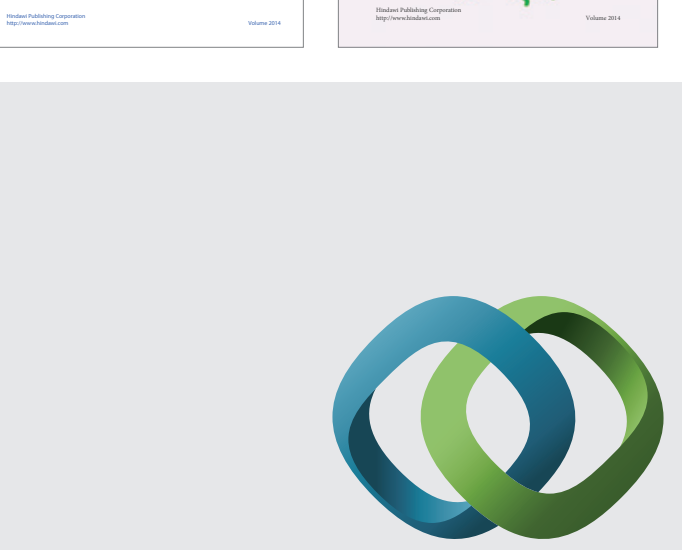

\section{Hindawi}

Submit your manuscripts at

http://www.hindawi.com
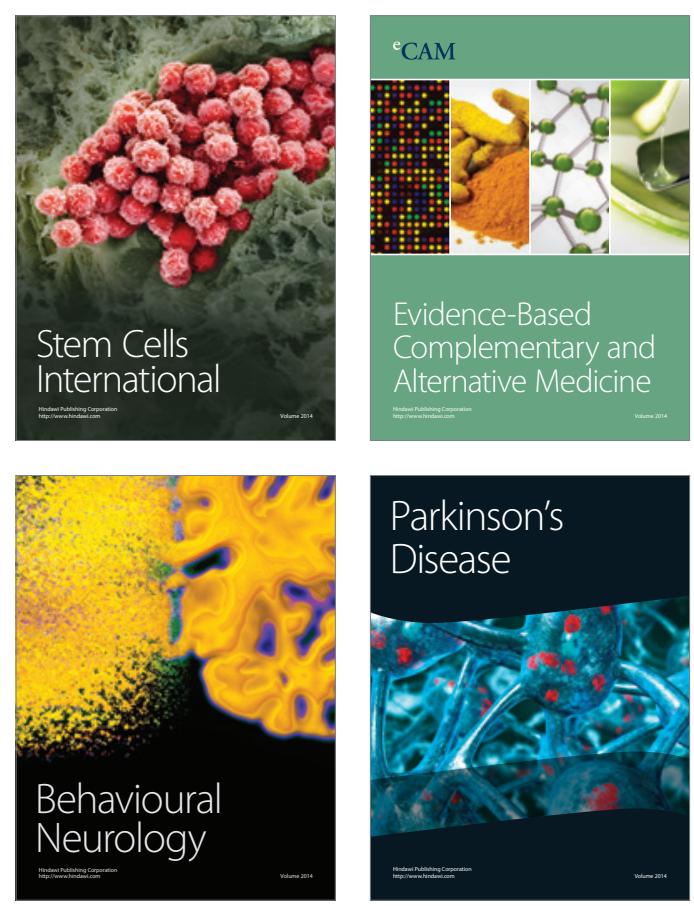

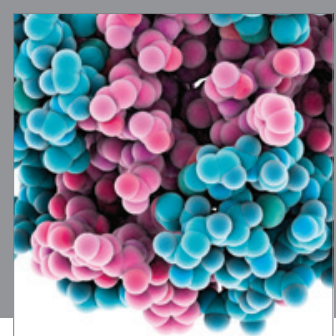

Journal of
Diabetes Research

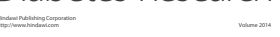

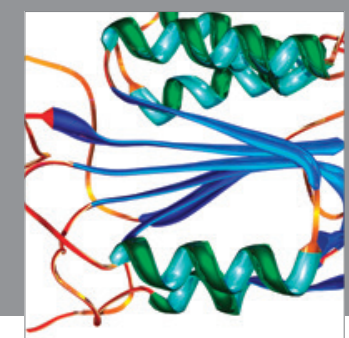

Disease Markers
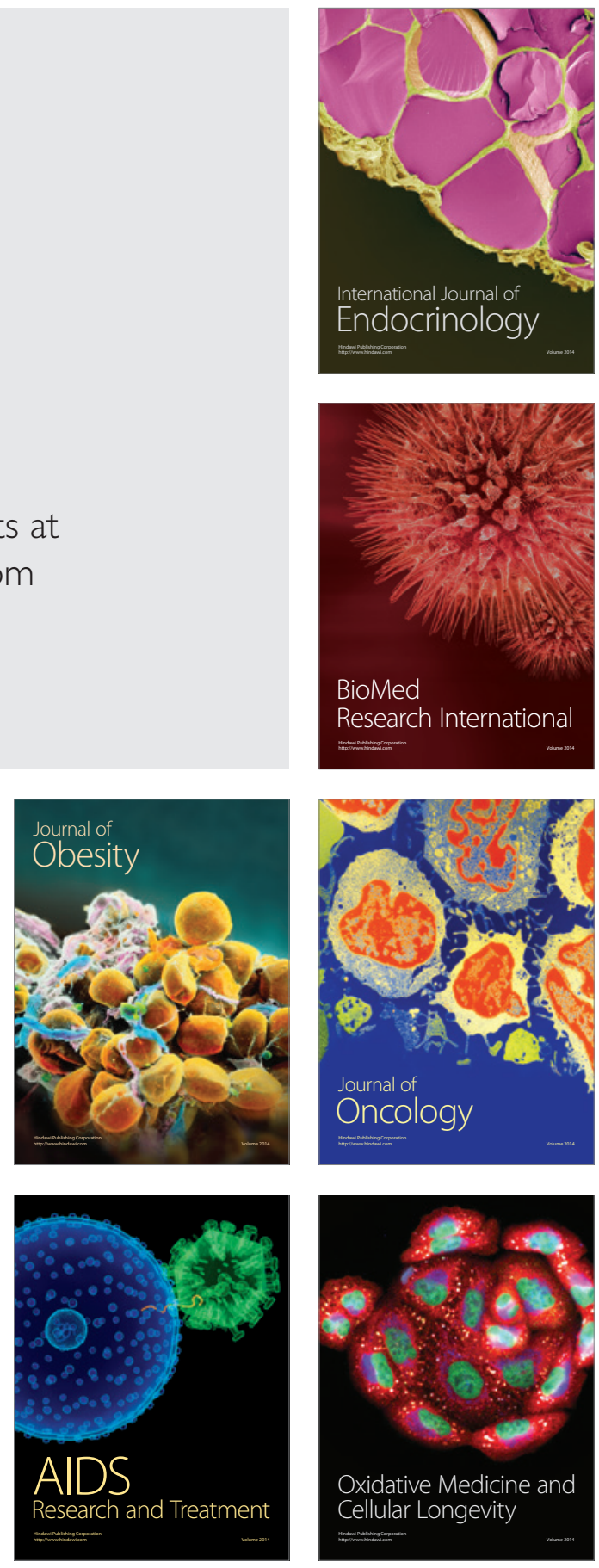